\title{
openheart Management principles in patients with COVID-19: perspectives from a growing global experience with emphasis on cardiovascular surveillance
}

\author{
Gruschen R Veldtman (D) , ${ }^{1}$ Mario Pirisi, ${ }^{2}$ Enrico Storti, ${ }^{3}$ Asad Roomi, ${ }^{4}$ \\ Fadl Elmula M Fadl-Elmula, ${ }^{5}$ Olga Vriz, ${ }^{6}$ Sabahat Bokhari, ${ }^{7}$ Naser Ammash, ${ }^{8}$ \\ Yezan Salam (D) , 9 Guang Zong Liu, ${ }^{10}$ Stefano Spinelli, ${ }^{11}$ Greta Barbieri, ${ }^{11}$ \\ Shahrukh Hashmi ${ }^{12}$
}

To cite: Veldtman GR, Pirisi M, Storti E, et al. Management principles in patients with COVID-19: perspectives from a growing global experience with emphasis on cardiovascular surveillance. Open Heart 2020;7:e001357. doi:10.1136/ openhrt-2020-001357

GRV and ES contributed equally.

Received 6 June 2020 Revised 7 August 2020 Accepted 19 August 2020

\section{D) Check for updates}

(c) Author(s) (or their employer(s)) 2020. Re-use permitted under CC BY-NC. No commercial re-use. See rights and permissions. Published by BMJ.

For numbered affiliations see end of article.

Correspondence to Dr Gruschen R Veldtman; gruschen@me.com

\section{ABSTRACT}

The COVID-19, due to SARS-CoV-2, has uncovered many real-world issues when it comes to healthcare management and has led to a widespread mortality. Observations thus far from the reports of COVID-19 have indicated that certain risk groups for example, those with pre-existing cardiovascular (CV) disease, hypertension, diabetes, chronic kidney disease and tobacco use are prone to disease development and specifically development of severe disease and possible fatality. It is increasingly evident that many CV conditions occur frequently. These include myopericarditis, acute coronary syndromes, thrombosis, arrhythmias, hypertension and heart failure. Many professional organisations and societies related to cardiology have produced guidelines or recommendations on most of the above-mentioned aspects. Given these rapid developments, the aims of this review manuscript were to summarise and integrate recent publications with newly developed guidelines and with the first-hand experience of frontline physicians and to yield a pragmatic insight and approach to CV complications of COVID-19. We emphasise on a strategic tier-based approach for initial assessment and management of COVID-19, and then delve into focused areas within CV domains, and additionally highlighting the role of point-of-care ultrasound especially lung ultrasound, echocardiography and electrocardiography, in the management of these patients. We hope this paper will serve as a useful tool in the CV management of COVID-19 for clinicians practicing in both developing and developed countries.

\section{BACKGROUND}

The pandemic of SARS-CoV-2 causing COVID$19^{1}$ has left healthcare providers overwhelmed and many fearful of the uncertainties inherent to effective healthcare provision. China and Italy were among the first countries which suffered from the effects of this pandemic ${ }^{2}$ and individuals involved in healthcare provisions have drawn important lessons and gained helpful insights into the pragmatic management of this pandemic. ${ }^{3-5}$
This review, which stems from the direct experience and expert opinion of cardiologists, internists and emergency physicians from different countries that were severely affected by COVID-19, aims to outline practical approaches for triaging, diagnosis, prognostication, systems redesign and then specifically focus on the most relevant cardiopulmonary effects of COVID-19 infection. Although many different professional organisations/societies from different regions have published recommendations/guidelines, we have consolidated those with the real-world experience of frontline physicians.

\section{METHODS}

We combined the first-hand knowledge and expertise acquired by clinicians on the frontline of COVID-19 crises in large volume centres, with a review of the literature and currently published guidelines. The process was via videoconferencing, discussion and debate of the key issues facing practitioners, and then subsequently through multiple revisions and updating in the light of new information. The coauthors were given the task of drafting a document that would be pragmatic and useful for the general clinicians dealing with the potential cardiovascular (CV) complications in patients with COVID-19.

\section{DISCUSSION AND OBSERVATIONS Pathophysiology}

SARS-CoV-2 utilises a transmembrane spike protein, also known as S-protein to bind to the host ACE2 receptor and 'eases' its way into the subcellular apparatus (figure 1). The process is complex, utilising conformational 


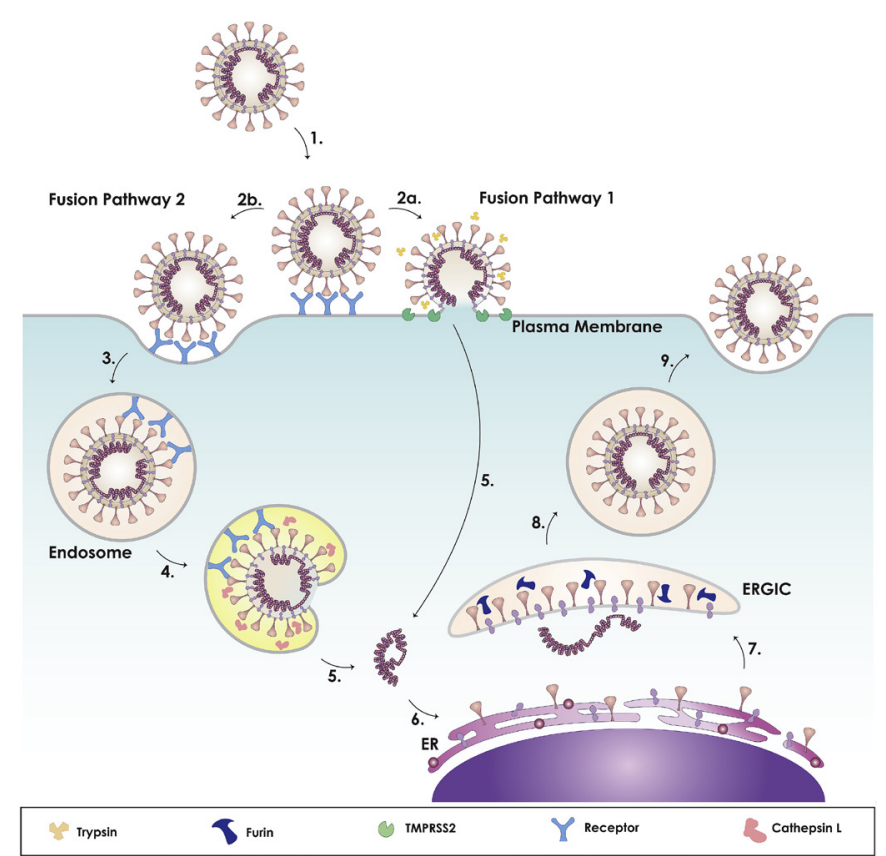

Figure 1 Model of coronavirus dual entry pathway. This model depicts the two methods of viral entry: early pathway and late pathway. As the virus binds to its receptor (1), it can achieve entry via two routes: plasma membrane or endosome. For SARS-CoV-2: the presence of exogeneous and membrane bound proteases, such as trypsin and TMPRSS2, triggers the early fusion pathway (2a). Oherwise, it will be endocytosed $(2 b, 3)$. For MERS-CoV: if furin cleaved the $S$ protein at S1/S2 during biosynthesis, exogeneous and membrane-bound proteases, such as trypsin and TMPRSS2, will trigger early entry (2a). Otherwise, it will be cleaved at the $\mathrm{S} 1 / \mathrm{S} 2$ site (2b) causing the virus to be endocytosed (3). For both: within the endosome, the low $\mathrm{pH}$ activates cathepsin $L$ (4), cleaving S2' site, triggering the fusion pathway and releasing the CoV genome. On viral entry, copies of the genome are made in the cytoplasm (5), where components of the spike protein are synthesised in the rough ER (6). The structural proteins are assembled in the ERGIC, where the spike protein can be precleaved by furin, depending on cell type (7), followed by release of the virus from the cell $(8,9)$. For SARS-CoV-2: studies currently show that SARS-CoV-2 can utilise membrane-bound TMPRSS2 or endosomal cathepsin L for entry and that the S protein is processed during biosynthesis. Other factors that can influence the viral entry pathway are calcium and cholesterol (not shown; from Tiffany Tang et $a^{60}$ ) ER, endoplasmic reticulum; ERGIC, ERGolgi intermediate compartment.

change of the S-protein itself into a puncture apparatus causing micropores in the host cell membrane.

\section{Clinical presentation and prognosis}

The clinical presentation of COVID-19 is highly variable ranging from asymptomatic or respiratory symptoms to refractory metabolic acidosis, coagulopathy/bleeding diathesis and/or fulminant respiratory failure with septic shock and multiorgan failure (MOF). Age, male gender ${ }^{6}$ and underlying comorbidities such as hypertension (HTN), diabetes mellitus, coronary artery disease (CAD), heart failure (HF), obesity, chronic kidney disease,
Box 1 Initial screening (Tier 1) survey for patients present to the emergency department with suspected COVID-19 infection

Level 1 : COVID-19 clinical survey

- History of flu-like symptoms.

- Fever at presentation.

- What is the $\mathrm{O}_{2}$ arterial saturation?

- Does the patient come from a known high-risk area or building or work environment or whether there is history of contact with patients with COVID-19 diagnosis.

- Comorbidities (hypertension, heart failure, coronary artery disease, diabetes mellitus, obesity, chronic lung disease, immunecompromised) or age $>60$.

chronic lung disease and tobacco use are reported to be the risk factors for poor outcomes. ${ }^{7}$

\section{Initial assessments}

Strategic, time-efficient and safe, (raining-based donning and doffing of personal protective equipment (PPE)) clinical assessment should be prioritised starting at the initial screening by the emergency department (ED) staff. A COVID-19 clinical survey (box 1) should be conducted by Tier-1 staff which will allow risk stratification for management.

Following the Tier-1 assessment, the patient can be transferred to a COVID-19 zone of an ED. Here patients should be triaged into those with mild, moderate or severe disease (box 2), and be subclassified into specific clinical phenotypes (table 1). It is essential to decide early which patients need high-level care or intensive care unit (ICU) monitoring. We have developed a simple algorithm to aid with this process (figure 2).

\section{Discharge from the ED}

Patients who have a clinically mild disease as outlined above and pass a simple brisk 50 to 100-metre walk test without dropping their arterial saturations on oximetry $3 \%-5 \%$ or more, can be considered fit for home discharge (but require home quarantine). ${ }^{8}$ This approach is outlined in the NHS guidance document on pulse oximetry screening. ${ }^{9}$ These patients need a clear set of instructions regarding signs of clinical deterioration including the development of fever, shortness of

\section{Box 2 Secondary screening (Tier 2) and triaging of patients} with confirmed or very likely COVID-19 infections

Level 2 : COVID-19 clinical survey

- Vital signs (temperature, blood pressure, heart rate, respiratory rate arterial saturations)

- Arterial blood gas

- Chest radiograph

- 6 min walk test

- Haemogram, C-reactive protein, rerritin, procalcitonin, renal panel, troponin

- COVID-19 PCR test 


\section{Table 1 Clinical phenotypic classification of COVID-19 infection}

\begin{tabular}{ll}
\hline Clinical phenotype classification & Type \\
\hline Mild disease &
\end{tabular}

Possible fever without respiratory failure, negative chest radiograph or 1

CT chest scan

\begin{tabular}{l} 
Moderate disease \\
$\begin{array}{l}\text { Possible fever with chest X-ray (or chest CT scan) positive for } \\
\text { consolidation and/or arterial blood gas analysis consistent with mild } \\
\text { respiratory failure }\end{array}$ \\
$\begin{array}{l}\text { Severe disease } \\
\text { Possible fever with severe respiratory failure evidenced by arterial blood }\end{array}$ \\
$\begin{array}{l}\text { gas analysis during triage } \\
\quad \begin{array}{l}\text { Respiratory failure with a suspicion of initial ARDS or complicated } \\
\text { pneumonia }\end{array}\end{array}$ \\
\hline ARDS
\end{tabular}

ARDS, acute respiratory distress syndrome.

breath and chest pain, and the use of telehealth clinics for follow-up has been very helpful. There is a growing awareness of a group of patients who experience so-called silent hypoxia, sometimes with saturations as low as $60 \%$ or less, without apparent shortness of breath. ${ }^{10}$ Performing a simple pulse oximetry test in all suspected or confirmed cases of COVID-19 is useful in detecting these individuals who may present in extremis if not detected in a timely fashion.

\section{Management strategies for effective inpatient treatment} Service reorganisation and redesignation

Of those admitted, the majority of patients with COVID-19 will have moderate disease. They can be cared for on dedicated COVID-19 wards fitted with specifically allocated equipment such as portable electrocardiographs, ultrasound machines and radiographic equipment specified for that clinical area. Each ward should have an area dedicated and equipped for donning/doffing of the PPE. Staff shifts, need prospective structuring to create 2-3 parallel teams, mitigating staff members becoming infected or those who require quarantine (figure 3)

Approximately $20 \%$ of the patients will eventually require admission to critical care areas. ${ }^{11}$ The majority of these will receive $\mathrm{O}_{2}$ by high-flow nasal cannulas or continuous positive airway pressure (CPAP) masks. The remaining will require intubation/ventilation. These ICUs may have a fivefold to tenfold increase in $\mathrm{O}_{2}$ demand which may precipitate supply shortage if not adequately planned. PPE shortage is a sobering reality that many institutions have had to deal with. In-hospital mortality is up to $25 \%$. This is anticipated to improve rapidly as the pathophysiology, risk factors, triaging, natural history and treatment strategies for COVID-19 are refined.

\section{Discharge from hospital}

Hospital stays are typically $5-14$ days for those admitted to medical wards, and 3-6 weeks for those who are admitted to the ICU. Discharge criteria include temperature resolution (for 48-72 hours at least), sustained arterial saturation $>93 \%$ in room air, heart rate pattern $<100$ beats per min and respiratory rate $<18$ breaths per minute. We do not believe it is always necessary to wait for complete resolution of symptoms or two consecutive negative nasopharyngeal swabs in the days before hospital discharge can be effected. For example when clinical testing is limited, a clinically recovered patient who did not have two negative reverse transcription polymerase chain reaction tests can still be discharged to self-isolate at home or in a safe place until resolution of fever for a minimum of 8 days for mild cases and 14 days for more severe cases. ${ }^{8}$ 
For patients in whom long-term recuperation is necessary, maintaining an oxygen saturation of $\geq 90 \%$ throughout a 6 min walk test may represent a feasible criterion to discharge the patient.

Diagnostics, inpatient surveillance and treatment approaches Daily clinical assessment and biomarkers

Clinical/laboratory testing should focus on detection of respiratory deterioration, as well as early detection of hyperinflammation syndromes. ${ }^{12}$ Routine laboratory examination includes haemogram with differential, blood cultures, coagulation profile, D-dimer, serum biochemical tests (including renal and liver function, creatine kinase, lactate dehydrogenase and electrolytes), myocardial enzymes, C-reactive protein (CRP), erythrocyte sedimentation rate, ferritin, procalcitonin and pro-brain natriuretic peptide. In our experience, and concordant with the literature, lymphopaenia, D-dimer elevation, increasing lactic acid levels and IL-6 derangements, and progressive chest imaging findings, are the most powerful prognostic markers for poor outcomes including the need for intubation, ARDS and death. ${ }^{13-15}$

\section{Escalation of inpatient treatment to ICU}

It is expected that up to $14 \%$ of infected individuals will have severe disease and potentially require escalation of care to critical care facilities. Older age, frailty, family wishes, comorbidities, arterial $\mathrm{O}_{2}$ saturation, respiratory rate, respiratory mechanics, $\mathrm{PO}_{2} / \mathrm{FiO}_{2}<150$, blood gas analysis, non-invasive mechanical ventilation trial response, chest X-ray (CXR), chest CT scan, lung ultrasound (LUS), degree of lymphopaenia, D-dimer level and troponin levels should be factors used in the decision-making process for such escalation of care. For example, in the elderly and those with significant frailty, patients themselves or the family may desire only supportive measures such as supplemental or high-flow nasal cannula $\mathrm{O}_{2}$ and not intubation and ventilation. In the presence of worsening respiratory distress and evolving ARDS, careful attention to $\mathrm{PO}_{2} / \mathrm{FiO}_{2}$, progressive multiorgan compromise, biomarker profiles as indicated above, should prompt the institution of critical care management. ${ }^{16}$ Many institutions may be overwhelmed by sudden increases in numbers and novel solutions to broadening capacity have been developed. ${ }^{17}$

\section{Chest imaging}

Wang $e a^{18}$ reported their experience on temporal changes in chest CT findings in a total of 366 chest CT scans done in 90 patients. ${ }^{18}$ The predominant pattern of abnormality was ground-glass opacity followed by consolidation. The predominant predischarge CT (median days of disease 24) pattern was ground-glass opacity in $60 \%$ of the patients in this study (figure 4). This is a rapidly evolving field and currently many machine-learning based strategies are being employed for specific diagnosis of COVID-19 pneumonia. Common CXR findings were similar to those described in CT scan: bilateral, peripheral consolidation and/or ground-glass opacities ${ }^{18}$ (figure 5).

\section{Cardiovascular surveillance}

Pre-existing CV disease defined the highest case fatality subgroup with $10.5 \%$ mortality, relative to $7.3 \%$ for diabetes,

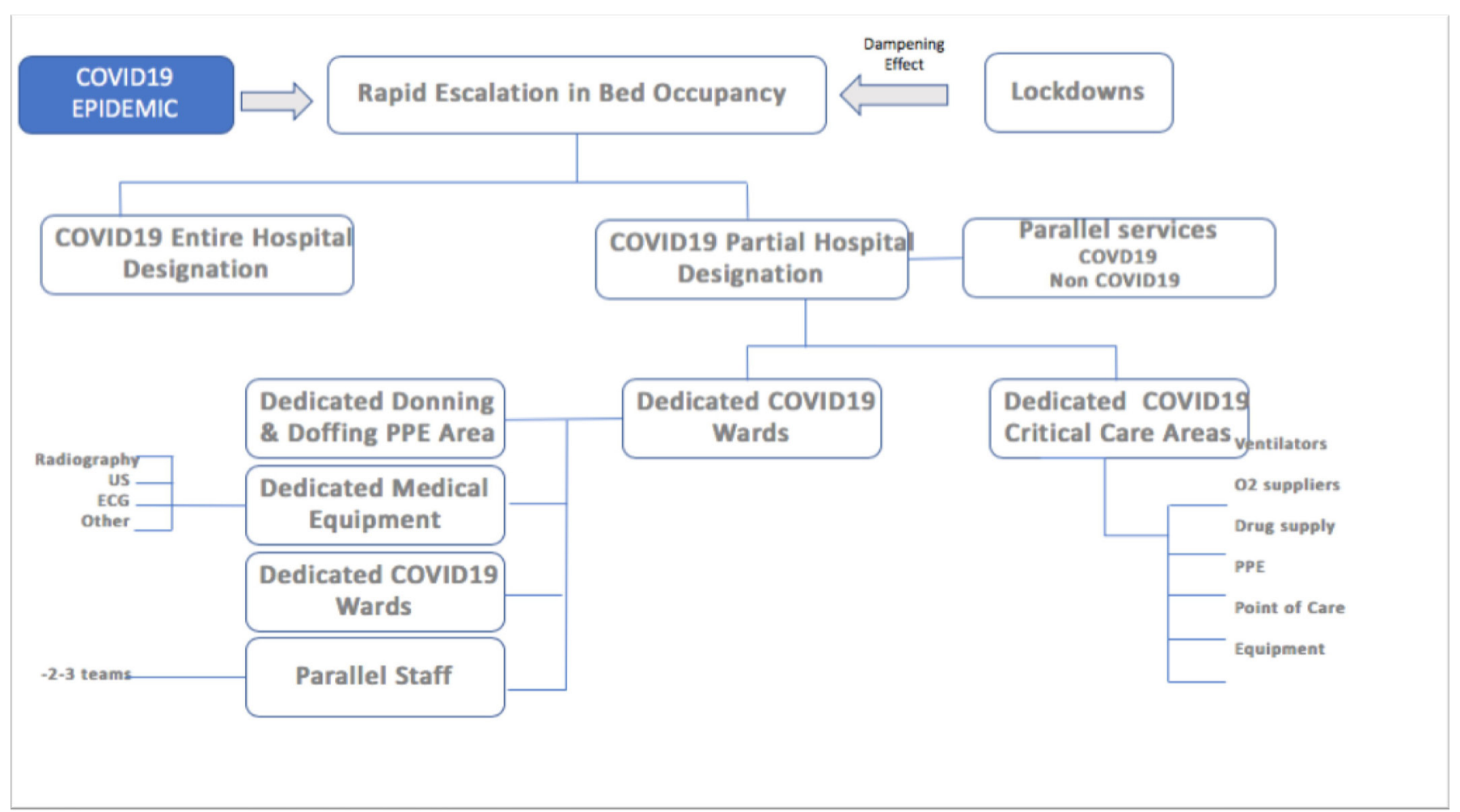

Figure 3 Systems oganisation and redesign. PPE, personal protective equipment. 


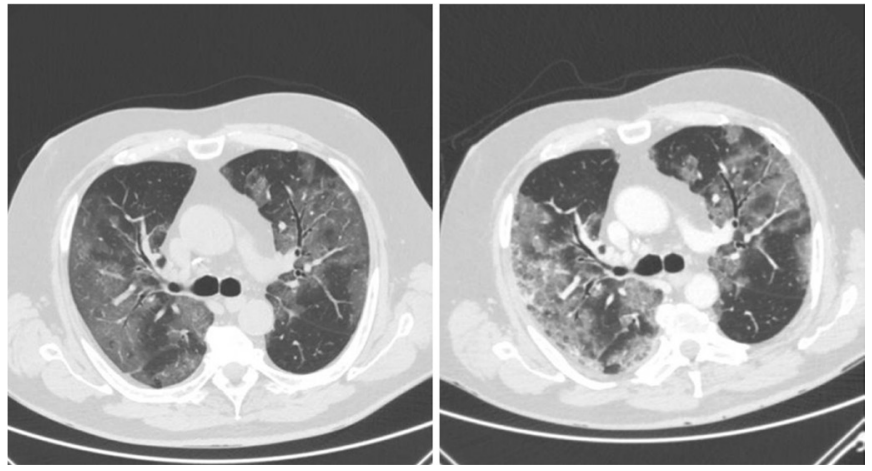

Figure 4 The most common CT features are demonstrated here in this clinical case presentation encountered by the coauthors: 62-year-old man presenting with fever, cough and dyspnoea. Multiple, largely confluent ground-glass opacities occupy more than half of both lungs. (B) Tn days later, ground-glass opacities have become more consolidated and a crazy-paving pattern has become evident, mostly at the periphery of the right lung.

$6.3 \%$ for chronic respiratory disease, $6.0 \%$ for HTN and $5.6 \%$ for cancer in a report. ${ }^{19}$ Sudden cardiac death was documented in $3 \%$ of COVID-19 infections. ${ }^{20}$ We propose a simple approach for clinicians for CV surveillance below, and we summarised the approach in table 2.

\section{Focal myocardial injury}

Patients with CAD may be at particular risk as a result of coronary plaque rupture secondary to viral-induced inflammation. Procoagulant effects of systemic inflammation may also increase the likelihood of stent thrombosis in those with a history of previous coronary intervention.

\section{Diffuse myocardial injury originating from generalised processes}

During the cytokine storm in COVID-19, a systemic inflammatory response syndrome occurs which can potentiate generalised organ damage including the heart. Murine models and human autopsy samples demonstrate that SARS-CoV-2 can downregulate myocardial and pulmonary ACE2 pathways, ${ }^{21}$ thereby mediating myocardial inflammation, lung oedema and acute respiratory failure.

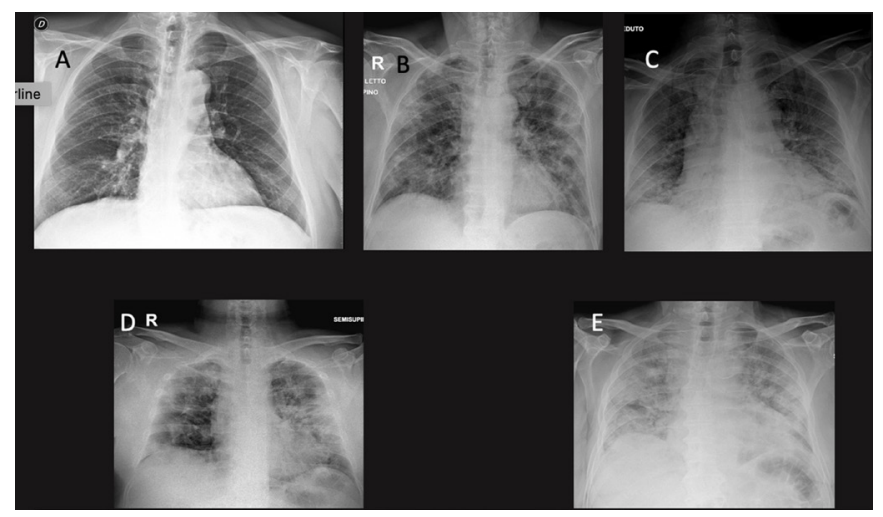

Figure 5 Progressively severe $X$-ray changes seen in COVID-19 in clinical scenarios encountered by the coauthors. $5 \mathrm{E}$ demonstrates fulminant acute respiratory distress syndrome.

\section{Stress cardiomyopathy}

Stress cardiomyopathy is another manifestation of myocardial injury occurring in patients with COVID-19. The stress induced by the pandemic, or the stress of the diagnosis or potentially also the physiologic consequences of infection in the patient, may trigger extreme sympathetic stimulation and microvascular constriction. Increasingly case reports are being reported particularly in older patients with typical echocardiographic features of left ventricular apical dyskinesia and ballooning, and basal hypercontractility and reduced ejection fraction. $^{22}$

\section{Troponin elevation syndromes}

Up to $12 \%$ of inpatients with COVID-19 have highly sensitive troponin (hs-Tn) elevation which is highly correlated with inflammatory biomarkers such as CRP, and procalcitonin. $^{23}$ hs-Tn elevation is a very sensitive marker of COVID-19 with reports of HF and arrhythmia in up to $60 \%$ of the patients in one series, ${ }^{24}$ and a mortality of up to $38 \%$. When hs-Tn elevation occurs in the presence of pre-existing CV disease, mortality may be higher as $69 \%$. Serial elevation in hs-Tn also is an important biomarker for subsequent mortality. ${ }^{15}$

\section{Treatment strategies}

Troponin elevations should drive supportive measures such as treatment of cytokine responses, ${ }^{12} 25$ secondary infection control and reevaluation of the thromboprophylaxis strategy. Only in the presence of chest pain, appropriate reciprocal ECG changes and evolving enzymes should standard approaches to acute coronary syndrome (ACS) be undertaken. Patients with ACS should receive statins as per standard recommendations, however, certain antivirals given for treatment of COVID-19 for example, Lopinavir/Ritonavir affect the CYP3A which can lead to severe drug interactions, and should be either avoided or used with extreme caution.

\section{Myopericarditis}

Fulminant myocarditis/cardiomyopathy has been documented in COVID-19 infections. ${ }^{26}$ Manifestations include hypotension, myocardial hypertrophy and dysfunction, marked elevation in cardiac enzymes and ECG changes (ST elevation and T-wave changes), and MRI evidence of myocardial inflammation. Ruan et al reported 5 of 150 patients with arrhythmia and deaths related to presumed severe myocarditis. ${ }^{27}$ Though direct viral localisation within myocardial tissue has been documented, the local pathological mechanisms have as yet not been defined. ${ }^{29}$

\section{Treatment strategies}

Rescue therapeutic approaches in moderate/severe cases can be used including immunoglobulins, corticosteroids and mechanical circulatory support if needed. Continuous cardiac rhythm monitoring is necessary. Impending HF should be diagnosed and treated early. In those with poor myocardial function, intracardiac thrombus should 


\begin{tabular}{|c|c|c|c|}
\hline Method & In whom and how often & Potential findings & Significance \\
\hline ECG & $\begin{array}{l}\text { Baseline in all with evidence of moderate } \\
\text { or severe COVID-19 infection } \\
\text { Any with pre-existing CV disease or high- } \\
\text { risk factor burden } \\
\text { Those with arrhythmia } \\
\text { Marked cTn elevation } \\
\text { Typical ACS symptoms } \\
\text { Daily in those given hydroxychloroquinexr }\end{array}$ & $\begin{array}{l}\text { ST-segment and T-wave changes } \\
\text { Reciprocal changes } \\
\text { Arrhythmias } \\
\text { Prolonged QT }\end{array}$ & $\begin{array}{l}\text { Coronary angiography in those with reciprocal } \\
\text { ECG changes and hs-Tn elevation particularly } \\
\text { if progressive }\end{array}$ \\
\hline $\begin{array}{l}\text { Transthoracic } \\
\text { echocardiography } \\
\text { Point-of-care approach } \\
\text { useful (POCUS) }\end{array}$ & $\begin{array}{l}\text { In those with moderate or severe } \\
\text { COVID-19 disease and cTn, BNP, } \\
\text { elevation or pre-existing disease } \\
\text { combination with high inflammatory } \\
\text { markers } \\
\text { Haemodynamic instability, suspicion } \\
\text { of left ventricular or right ventricular } \\
\text { dysfunction. } \\
\text { Pulmonary embolism } \\
\text { Dedicated echocardiography in } \\
\text { significantly elevated troponin or ECG } \\
\text { abnormalities and/or concern for } \\
\text { congestive HF }\end{array}$ & $\begin{array}{l}\text { Left ventricular dysfunction (diffuse or } \\
\text { segmental) } \\
\text { Increased wall thickness } \\
\text { (pseudohypertrophy-myocarditis) } \\
\text { Intracardiac thrombosis } \\
\text { Pericardial effusion } \\
\text { Reduced TAPSE and right ventricular } \\
\text { function } \\
\text { Elevated right ventricular systolic } \\
\text { pressures, Inferior vena caval distension } \\
\text { without respiratory variation (not true for } \\
\text { patients in ventilation) } \\
\text { - Raised right ventricular filling pressures } \\
\text { Evidence of stress cardiomyopathy }\end{array}$ & $\begin{array}{l}\text { Focused echocardiography should be } \\
\text { performed only in those where management } \\
\text { likely to be influenced } \\
\text { Limited studies to address specific } \\
\text { clinical questions } \\
\text { Left ventricular hypertrophy and left } \\
\text { ventricular dysfunction and pericardial } \\
\text { effusion may indicate myocarditis } \\
\text { Combination of LUS congestion + high } \\
\text { right ventricular filling pressures may } \\
\text { signal imminent CV decline; consider ICU } \\
\text { admission/intubation }\end{array}$ \\
\hline $\begin{array}{l}\text { MRI } \\
\text { criteria for inflammation } \\
\text { or injury } \\
\text { (Lake Louise Consensus } \\
\text { Criteria) }\end{array}$ & $\begin{array}{l}\text { Those with inconclusive clinical } \\
\text { and echocardiographic evidence of } \\
\text { myocarditis }\end{array}$ & $\begin{array}{l}\text { T1 and T2 -mapping sequences and T1 } \\
\text { inversion recovery (STIR) sequences } \\
\text { for myocardial interstitial oedemaLate } \\
\text { gadolinium enhancement can be } \\
\text { considered }\end{array}$ & $\begin{array}{l}\text { MRI may help confirm diagnosis in } \\
\text { suspected or exclude in doubtful } \\
\text { myocarditis patients, stress } \\
\text { cardiomyopathy }\end{array}$ \\
\hline Endomyocardial biopsy & $\begin{array}{l}\text { Role not clearly defined yet } \\
\text { Differentiating direct viral myocardial } \\
\text { involvement from secondary causes }\end{array}$ & $\begin{array}{l}\text { New-onset HF of less than } 2 \text { weeks' } \\
\text { duration associated with a normal- } \\
\text { sized or dilated left ventricle and } \\
\text { haemodynamic compromise } \\
\text { Presence of viral particles within } \\
\text { myocardium }{ }^{29}\end{array}$ & $\begin{array}{l}\text { Identification of direct viral localisation in } \\
\text { the myocardium } \\
\text { Identification of secondary immune } \\
\text { effects } \\
\text { - Assessment of response to drug therapy }\end{array}$ \\
\hline
\end{tabular}

ACS, acute coronary syndrome; BNP, brain natriuretic peptide; cTn, cardiac troponins; CV, cardiovascular; HF, heart failure; hs-Tn, highly sensitive troponin; ICU, intensive care unit; LUS, lung ultrasound; POCUS, point-of-care ultrasound; TAPSE, tricuspid annular plane systolic excursion.

be suspected, and if confirmed, therapeutic anticoagulation commenced unless contraindicated.

\section{Arrhythmias and ECG abnormalities}

Arrhythmias may complicate hospitalised patients with COVID-19 in up to $16.7 \%$ of cases and tend to prolong the ICU stay. The arrhythmia mechanisms are highly variable and include supraventricular tachycardia, polymorphic ventricular tachycardia (VT) and torsade 3031 (figure 6). The exact aetiology of arrhythmias is unclear at present, but besides the usual culprits (eg, hypoxia, inflammatory cascade, myocarditis), drug therapy such as chloroquine, hydroxychloroquine, moxifloxacin and azithromycin can also be aetiologic factors. ECG abnormalities observed in clinical practiced are summarised in box 3 and figure 6 .
Treatment strategies

A number of comprehensive COVID-19-related arrhythmia guidelines have recently been published, and we summarise them in table $3 \mathrm{~A} \mathrm{~B} .{ }^{3233}$

\section{Patients on antihypertensive medications}

The ACE2 receptor, though sharing some homology with $\mathrm{ACE}$, is a distinct transmembrane protein which does not convert Ang-1 to Ang-2 and is used by SARS-CoV-2 to gain cell entry. ACE inhibitors and angiotensin receptor blockers do not directly inhibit ACE2, but in decreasing levels of Ang-1 and Ang-2, can potentially result in upregulation of ACE2, hence there is a concern about possible potentiation of viral entry. ${ }^{34}$ These elevations in transmembrane ACE2 expression may mediate anti-inflammation, antiapoptotic and antiproliferative 

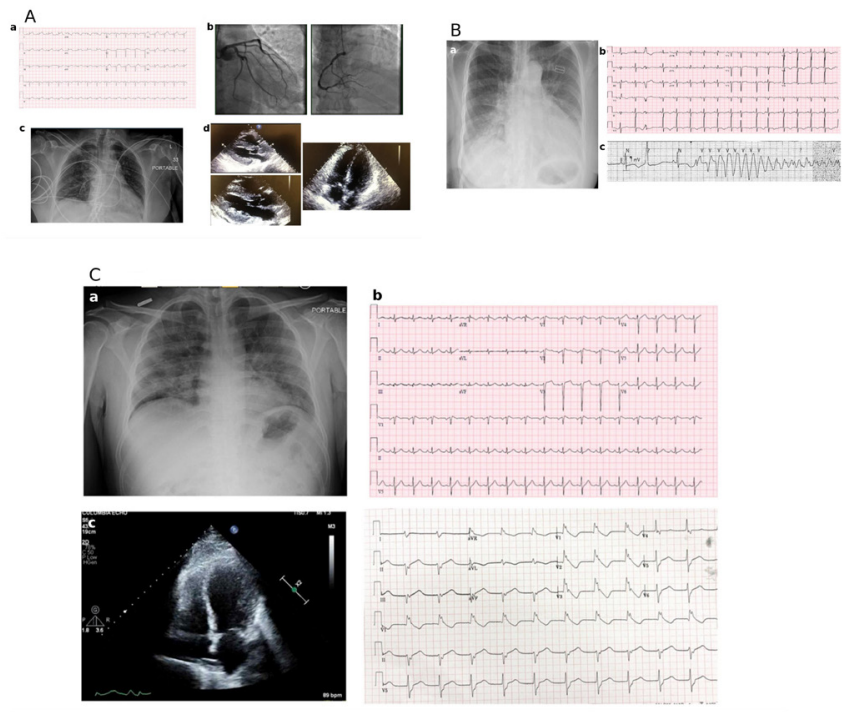

Figure 6 (A) Various ECG abnormalities seen in patients with COVID-19 (from Fried et $a /^{30}$ ). Chest pain and ST elevation. Initial ECG showed sinus tachycardia, lowvoltage QRS complexes in the limb leads and diffuse ST elevation in leads I, II, aVL and leads V2-V6 (a). Coronary angiogram demonstrated mild disease in the left anterior descending artery and left circumflex artery and $40 \%$ stenosis in the mid-right coronary artery (b). Chest radiography demonstrated clear lungs (c). Transthoracic echocardiogram with severe increased left ventricular wall thickness and left ventricular ejection fraction approximately $30 \%$ with trace circumferential pericardial effusion (d). (B) Decompensated HF. Chest radiography shows pulmonary vascular congestion, patchy airspace opacities at bases and bilateral pleural effusions (a). ECG shows sinus rhythm with premature atrial and ventricular complexes, lateral T-wave inversions, and a prolonged QT interval (b). Telemetry strip shows prolonged QT interval and torsades de pointes after R-on-T phenomenon (c). (C) Cardiogenic shock rescued by veno-arterial-venous extracorporeal membrane oxygenation. Chest radiograph showed diffuse ill-defined airspace opacities bilaterally (a). Initial ECG (top) demonstrated sinus tachycardia with incomplete right bundle-branch block. Repeat ECG (bottom) demonstrated accelerated idioventricular rhythm (b). Transthoracic echocardiogram demonstrated left ventricular end-diastolic diameter of $4.5 \mathrm{~cm}$, left ventricular ejection fraction $20 \%$ to $25 \%$, with akinesis of mid-left ventricular segments (c). HF, heart failure.

effects through ACE2/MAS/Angiotensi1-7 pathways. ${ }^{34} 35$ In our experience from China (GZL), we found that the COVID-19 infection did not cause significant increases in blood pressure, however, some patients had slightly elevated blood pressure due to anxiety.

Indeed these early observations were confirmed in new studies assessed in two new meta- analyses ${ }^{36}{ }^{37}$ which demonstrated no significant increases urinary or serum ACE2 levels, and demonstrated there was not greater mortality or greater disease severity with exposure to ACE inhibitor of ARB's. Some protective effect has been demonstrated on mortality outcomes in those with HTN controlled with these agents.

\section{Box 3 Varying ECG abnormalities observed}

Rhythm abnormalities
Ventricular tachycardia
Torsades de pointes
Atrial fibrillation
Supra ventricular tachycardia
Specific abnormalities
ST elevation in I, II, aVL and V2-6. Coronary angiography demon-
strated non-occlusive coronary artery disease
Sinus tachycardia in patients with cardiogenic shock and severe left
ventricular dysfunction
Premature atrial and ventricular complexes, lateral-wave inversion
and prolonged QT
Sinus rhythm with non-specific ST and T-wave changes

\section{Treatment strategies}

As a general rule, antihypertensive medication(s) should not be stopped in patients with COVID-19 unless another compelling reason such as hypotension is evident.

\section{Point-of-care ultrasound (POCUS)}

POCUS is a rapidly accessible and economic bedside screening tool, and can help identify the various complications of COVID-19 infections such as deep vein thrombosis (DVT), pneumonia and myocarditis. ${ }^{38}$ LUS has demonstrated reassuring correlation with the gold standard chest CT in detecting COVID-19 pneumonia. ${ }^{39} 40$ LUS can identify pulmonary parenchymal alterations commonly seen in patients with COVID-19 and can identify progressive degrees of loss of lung aeration, indicative of disease worsening. ${ }^{41}$ Typical lung alterations include multiple B-lines with a patchy distribution, irregular pleural line, peripheral small consolidations and sometimes, especially in more advanced phases, larger consolidations with tissue-like appearance ${ }^{42}$ (box 4; figure 7).

LUS is currently being used by us in the following settings with appropriate clinical contextualisation with great effect:

1. ED: to detect pulmonary involvement with a high sensitivity and negative predictive value. Thus it is superior to CXR in this regard.

2. Inpatient monitoring: to detect progressive pulmonary involvement with loss of aeration and risk stratify patients.

3. Severe patients: to monitor response to interventional lung therapies such as continuous positive airways pressure, mechanical ventilation, prone ventilation and alveolar recruitment manoeuvres.

Specific benefits we have personally experienced from using LUS include among others identification of false-negative viral PCR testing when the LUS showed typical features in the presence of suspicious symptoms and using LUS in concert with other clinical respiratory characteristics. The latter has allowed early detection of those likely to deteriorate. LUS limitations include 
Table 3 (A) Table outlining strategies to limit arrhythmia risk and burden in patients with COVID-19. (B) Emergent or urgent indications for electrophysiology procedures during COVID-19

\section{Strategies to minimise arrhythmia risk in patients with COVID-19}

Withhold drugs associated with QTc prolongation in those with baseline QTC $\geq 500 \mathrm{msec}$ OR with known long QT syndromes (eg, for nausea/vomiting in an intensive care unit patient, avoid ondansetron)

Monitor cardiac rhythm and QT interval, withdrawing QT prolonging drugs if QTC $>500 \mathrm{msec}$

Correct hypokalaemia to levels of $>4 \mathrm{mEq} / \mathrm{L}$ and hypomagnesaemia to $>2 \mathrm{mg} / \mathrm{dL}$, and keep calcium levels in the normal range

Avoid other QTc prolonging agents where possible

Drugs/agents used in patients with COVID-19 (for specific treatment or supportive care) that are associated with QT prolongation, VT, VF, LQT

- Chloroquine (proposed as an antiviral treatment)

- Hydroxychloroquine (proposed as an antiviral treatment)

- Lopinavir/Ritonavir (proposed as an antiviral treatment)

- Azithromycin (proposed as an antiviral treatment)

- COVID-Organics herbal tea (artemisia derived; projected as an antiviral treatment)

- Nigella Sativa (projected as an antiviral treatment)

- Citalopram, escitalopram and sertraline (used for depression symptoms in patients with COVID-19; increased use in general public during the pandemic)

- Fluoroquinolones (used commonly in patients in ICU with confirmed COVID-19 pneumonia)

- Miscellaneous: alcohol or grapefruit ingestion can interact with certain medications and increase the risk of arrhythmias

\section{HRS-ACC-AHA ESC}

1. VT ablation for medically uncontrolled electrical storm in a hemodynamically Catheter ablation: compromised patient

2. Catheter ablation of incessant, hemodynamically significant, severely symptomatic tachycardia not responding to antiarrhythmic drugs, rate control and/or cardioversion

3. Catheter ablation for Wolff Parkinson White syndrome or pre-excited Atrial Fibrillation with syncope or cardiac arrest

4. Lead revision for malfunction in a pacemaker-dependent patient or patient on ICD receiving inappropriate therapy

5. Generator change in pacemaker-dependent patients who are at ERI or at device EOL

6. Pacemaker or ICD generator change with minimal battery remaining, depending on specific clinical situations

7. Secondary prevention ICD

8. Pacemaker implant for complete heart block, Mobitz II AVB, or high grade AVB with symptoms or severe symptomatic sino-atrial node dysfunction with long pauses

9. Lead/device extraction for infection, including patients not responding to antibiotics or for endocarditis, bacteremia or pocket infection

10. Cardiac resynchronisation therapy in the setting of severe refractory heart failure in guideline indicated patients

11. Cardioversion for highly symptomatic atrial arrhythmias or rapid ventricular rates not controlled with medications

12. Transesophageal echocardiography for patients who need urgent cardioversion

AF, atrial fibrillation; AVB, atrioventricular block; CIED, cardiac implantable electronic devices; EOL, End Of Life; ERI, elective replacement indicator; ICD, implantable cardiac defibrillator; LQT, long QT; VT, ventricular tachycardia.

operator dependence, lack of guidelines on standardised methodology, the inability to detect deep lung lesions which do not reach the pleural line and a general lack of specificity. ${ }^{39} 43$ Gargani et al recently reviewed our clinical experience and approach with LUS in patients with COVID-19. ${ }^{44}$

\section{Echocardiography and Doppler US}

POCUS and focused echocardiography are already part of many ICU's and high dependency areas. ${ }^{45} 46$ Pointof-care echocardiography can identify left and right ventricular dysfunction, intracardiac thrombus, high filling pressures particularly on the right side as indicated by a distended inferior vena cava that does not
1. VT/NF ablation for electric storm

2. AF causing syncope or tachycardiomyopathy

3. WPW syndrome with fast pre-excited AF and/or syncope and/or cardiac arrest

CIED:

1. Urgent pacemaker implantation for symptomatic high-degree AVB or sinus node dysfunction with long asystolic pauses

2. Urgent secondary prevention ICD implantation for cardiac arrest or VT

3. ICD/pacemaker battery replacement for imminent or actual EOL in pacemaker dependent patients

4. Lead extraction for infection

5. Lead revision for symptomatic malfunction

Cardioversion/other electrophysiology procedures:

1. Highly symptomatic, medically refractory atrial fibrillation/ atrial flutter collapse with inspiration, and elevated right ventricular systolic pressures. ${ }^{47}$ Doppler US and compression of the femoral/popliteal veins when limb oedema is present can help early detection of DVT. ${ }^{48}$ At present, there is a minimal role for the use of transesophageal echocardiography.

\section{Integrated cardiopulmonary ultrasound}

In the case of a patient with right ventricular dysfunction, inferior caval vein dilation \pm associated left ventricular dysfunction and pulmonary parenchymal lesions (such as ground glass lesions), detected by integrated mechanical ventilation including intubation has to be considered. $^{49}$ 
Box 4 Commonly detected abnormality on lung ultrasound in COVID-19 respiratory infections

\section{Abnormality}

- Varying degree of an interstitial syndrome (diffuse B-lines)

- Irregular pleural line

- Small subpleural lung consolidations

- Larger consolidations less frequent

- Small localised pleural effusion

Signs of worsening lung involvement are

- Progression towards white lung

- From white lung to lung consolidation

Increase in the number of lung segments with coalescent B-lines

Extension of B-lines to the anterior lung segment

\section{Thrombotic burden and anticoagulation strategies}

Patients with COVID-19 have thrombi and microthrombi. The frequency is likely underestimated and depends on the setting that is, critical care versus inpatient versus outpatient, screening strategy and screening modality. When twice daily ultrasound was used in the ICU setting frequencies of DVT of up to $85 \%$ have been documented.$^{50}$ The 'epicentre' of the coagulopathy is believed to be the lung where intra-alveolar fibrin production and its degradation is believed to contribute to endothelial dysfunction and dysregulation of the haemostatic system. ${ }^{51}$ The cascade of haemostatic abnormalities has been named COVID-19 Associated Hemostatic Abnormality or CAHA. ${ }^{51-53}$ The spectrum of CAHA varies from

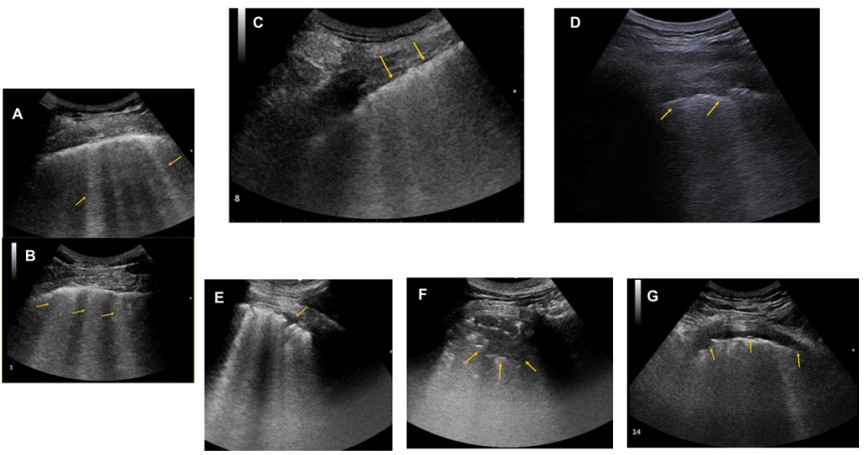

Figure 7 Key LUS phenomena in COVID-19. The relevant feature on each image are outlined by the yellow arrows. (A) Separated B-lines - the figure shows some separated Blines. (B) Coalescent B-lines-the figure shows coalescent B-lines without frank alterations of the pleural line. (C) White lung-the figure shows many coalescent B-lines, generating a sonographic 'white lung' appearance. (D) Irregular pleural line-the figure shows an irregular 'fragmented' pleural line with some B-lines. (E) Small peripheral consolidationthe figure shows a small peripheral consolidation in the context of multiple B-lines with an irregular pleural line. $(F)$ Larger consolidation - the figure shows a large tissue-like consolidation with air-bronchogram. (G) Localised trivial pleural effusion: the figure shows a trivial pleural effusion (anechoic space between the parietal and visceral pleural layer), with an irregular pleural line and B-lines. LUS, lung ultrasound. twofold to threefold elevation in D-dimers, seen in mild infection, to more than sixfold increases in D-dimer and disseminated intravascular coagulation (DIC). In a study of 183 patients with COVID-19 with severe pneumonia, markedly elevated D-dimer and fibrinogen degradation products were associated with an $11.5 \%$ overall mortality. Seventy-four per cent of non-survivors and $0.6 \%$ of survivors met the criteria of DIC. ${ }^{54}$ Klok et al reported a $31 \%$ incidence of thrombotic complications in COVID-19 affected patients in ICU ( $\mathrm{n}=183)$, pulmonary embolism being the most common. ${ }^{52}$ Elevated D-dimer $\geq 1.5 \mathrm{ug}$ / $\mathrm{mL}$ demonstrated a high predictive value for the subsequent development of venous thrombo embolism (VTE) ${ }^{55}$ Admission D-dimer levels $>2.0 \mu \mathrm{g} / \mathrm{mL}$ (fourfold increase) accurately predicted in-hospital mortality, using a biomarker for morbid and mortality outcomes. ${ }^{56}$

\section{Treatment approaches}

Given these risks, standard thromboprophylaxis with low-molecular-weight heparin (LMWH) or fondaparinux should be considered unless contraindicated. Mechanical thromboprophylaxis remains controversial and guidelines differ on its universal implementation. An expert panel, on behalf of the International Society on Thrombosis and Haemostasis (ISTH),${ }^{57}$ has issued an interim guidance statement on the management of coagulopathy in patients with COVID-19 recommending the use of prophylactic dose LMWH unless there is active bleeding or a platelet count of $<25 \times 10^{9} / \mathrm{L}^{57}$ CHEST guidelines ${ }^{58}$ have recommended LMWH or fondaparinux over unfractionated heparin in the acutely ill hospital patient. Exact escalation indications for anticoagulation therapy to treatment dosing or higher levels remain controversial as the results of randomised controlled trails are awaited. Further, the bleeding risks associated with severe COVID-19 infection have not been clearly defined as yet. It is likely that recommendations therefore will evolve quickly and become patient specific. In general, patients with mild or moderate stable disease, and already on antiplatelet therapies or anticoagulant therapies (mechanical valves) such as warfarin or the direct oral anticoagulants, ought to continue these agents unless the haemorrhagic risk increases substantially. It is important to consider the potential for drug-drug interaction between COVID-19 investigational drugs such as lopinavir/ritonavir which, through inducing CYP2C9 and CYP1A2, may lower the international normalised ratio (INR). Similarly, antiarrhythmic drugs such as amiodarone and antibiotics may also affect the INR. In patients with pre-existing CAD on dual antiplatelet therapy post percutaneous coronary intervention, who develop COVID-19 infections, we advise the continuation of antiplatelets consistent with the underlying indication, especially if recent ACS or PCI, exceptions being severe thrombocytopaenia (ie, platelets $<50$ ), DIC or MOF. Thromboprophylaxis for 
atrial fibrillation should be guided by application of the CHA2DS2-VASc score. ${ }^{59}$

It is important to evaluate the need for post hospital discharge thromboprophylaxis. ISTH current guidelines recommend that it should be considered for all patients who meet high VTE risk criteria. ${ }^{57}$

\section{Depression among patients with COVID-19 infection}

In the current universal multimedia availability, fear for self and loved ones, anguish and depression are universally observed phenomena thereby prompt recognition and treatment of depression/anxiety is highly recommended. Communication between healthcare teams is of utmost importance and looking after the staff needs is necessary for a successful COVID-19 unit.

\section{CONCLUSIONS AND FINAL THOUGHTS}

During this pandemic, the patients with pre-existing CV disease are predisposed to complications of COVID-19; however, the infection itself or its treatments can lead to significant CV morbidity and mortality. A heightened suspicion for CV complications is necessary for optimum management of patients with COVID-19. Laboratory testing as outlined, POCUS, echocardiograms and electrocardiography tools should ideally be available in prompt diagnosis of these complications. In limited resource environments clinical acumen can still be the most effective in combination with relatively simple assessments of $\mathrm{P} / \mathrm{F}$ ratio, D-dimer, troponin, complete blood count and POCUS. Lastly, prospective data collection via well-designed epidemiologic studies and clinical trials are imperative in the ongoing battle against COVID-19.

\section{Author affiliations \\ ${ }^{1}$ Adolescent and Adult Congenital Heart Disease Program, King Faisal Specialist Hospital and Research Center, Riyadh, Saudi Arabia \\ ${ }^{2}$ Department of Translational Medicine, Università del Piemonte Orientale, Novara, Novara, Italy \\ ${ }^{3}$ Department of Critical Care, Maggiore Hospital, Lodi, Lodi, Italy \\ ${ }^{4}$ Department of Cardiology, Prince Sultan Cardiac Center, Riyadh, Riyadh, Saudi Arabia \\ ${ }^{5}$ Cardiology, Heart Centre, King Faisal Specialist Hospital and Research Centre, Riyadh, Riyadh, Saudi Arabia \\ ${ }^{6}$ Cardiology, King Faisal Specialist Hospital \& Research Centre, Riyadh, Saudi Arabia ${ }^{7}$ Cardiology, Columbia University, New York, New York, USA \\ ${ }^{8}$ Department of Cardiovascular Medicine, Mayo Clinic, Rochester, Minnesota, USA \\ ${ }^{9}$ College of Medicine, Alfaisal University, Riyadh, Riyadh Province, Saudi Arabia \\ ${ }^{10}$ Department of Cardiology, First Affiliated Hospital of Harbin Medical University, Harbin, Harbin, China \\ ${ }^{11}$ Emergency Medicine Unit, Pisa University Hospital, Pisa, Toscana, Italy \\ ${ }^{12}$ Clinical Trials Unit, King Faisal Specialist Hospital and Research Center, Riyadh, Saudi Arabia}

\section{Twitter Yezan Salam @ysalam1}

Acknowledgements We thank Dr Luna Gargani for her advice and expertise regarding the role of lung ultrasound in patients with COVID-19, and Dr Joseph Palumbo for his helpful input with respect to thrombosis and anticoagulation sections.

Contributors All authors have contributed to the manuscript with respect to its content, but specifically in the overall design, layout and broad messages contained in it. The physicians also agreed on the treatment approaches contained in the manuscript.

Competing interests SH has received honoraria from Pfizer, Novartis, Janssen and Mallinckrodt. SH has received travel grants from Gilead, Merck and Sanofi. Patient consent for publication Not required.

Provenance and peer review Not commissioned; externally peer reviewed.

Data availability statement As this is a review article, no datasets are available apart from the published literature.

Open access This is an open access article distributed in accordance with the Creative Commons Attribution Non Commercial (CC BY-NC 4.0) license, which permits others to distribute, remix, adapt, build upon this work non-commercially, and license their derivative works on different terms, provided the original work is properly cited, appropriate credit is given, any changes made indicated, and the use is non-commercial. See: http://creativecommons.org/licenses/by-nc/4.0/.

ORCID iDs

Gruschen R Veldtman http://orcid.org/0000-0002-9336-2662

Yezan Salam http://orcid.org/0000-0002-5611-6358

\section{REFERENCES}

1 Klement E, Godefroy N, Burrel S, et al. The first locally acquired novel case of 2019-nCoV infection in a healthcare worker in the Paris area. Clin Infect Dis 2020:ciaa171.

2 Onder G, Rezza G, Brusaferro S. Case-fatality rate and characteristics of patients dying in relation to COVID-19 in Italy. JAMA 2020. doi:10.1001/jama.2020.4683. [Epub ahead of print: 23 Mar 2020].

3 Lazzerini M, Putoto G. COVID-19 in Italy: momentous decisions and many uncertainties. Lancet Glob Health 2020;8:e641-2.

4 Spina S, Marrazzo F, Migliari M, et al. The response of milan's emergency medical system to the COVID-19 outbreak in Italy. Lancet 2020;395:e49-50.

5 Gagliano A, Villani PG, Co' FM, et al. COVID-19 epidemic in the middle Province of northern Italy: impact, logistics, and strategy in the first line Hospital. Disaster Med Public Health Prep 2020:1-5.

6 Grasselli G, Zangrillo A, Zanella A, et al. Baseline characteristics and outcomes of 1591 patients infected with SARS-CoV-2 admitted to ICUs of the Lombardy region, Italy. JAMA 2020. doi:10.1001/ jama.2020.5394. [Epub ahead of print: 06 Apr 2020].

7 Wu Z, McGoogan JM. Characteristics of and important lessons from the coronavirus disease 2019 (COVID-19) outbreak in China. JAMA 2020;323:1239.

8 ECDC. Guidance for discharge and ending isolation in the context of widespread community transmission of COVID-19 - first update. Available: https://www.ecdc.europa.eu/sites/default/files/documents/ covid-19-guidance-discharge-and-ending-isolation-first\%20update. pdf. https://www.ecdc.europa.eu/sites/default/files/documents/ covid-19-guidance-discharge-and-ending-isolation-first update.pdf. [Accessed 8 Apr 2020].

9 NHS. Pulse oximetry to detect early deterioration of patients with COVID-19 in primary and community care settings. publications approval reference: 001559. Available: https://www.england.nhs.uk/ coronavirus/wp-content/uploads/sites/52/2020/06/C0445-remotemonitoring-in-primary-care.pdf [Accessed 11 Jun 2020].

10 Teo J. Early detection of silent hypoxia in Covid-19 pneumonia using smartphone pulse oximetry. J Med Syst 2020;44:134.

11 Richardson S, Hirsch JS, Narasimhan M, et al. Presenting characteristics, comorbidities, and outcomes among 5700 patients hospitalized with COVID-19 in the new York City area. JAMA 2020. doi:10.1001/jama.2020.6775. [Epub ahead of print: 22 Apr 2020].

12 Mehta P, McAuley DF, Brown M, et al. COVID-19: consider cytokine storm syndromes and immunosuppression. Lancet 2020;395:1033-4.

13 Yang Y, Shen C, Li J, et al. Exuberant elevation of IP-10, MCP-3 and IL-1ra during SARS-CoV-2 infection is associated with disease severity and fatal outcome. medRxiv 2020.

14 Zhang C, Wu Z, Li J-W, et al. Cytokine release syndrome in severe COVID-19: interleukin-6 receptor antagonist tocilizumab may be the key to reduce mortality. Int J Antimicrob Agents 2020;55:105954.

15 Guo T, Fan Y, Chen M, et al. Cardiovascular implications of fatal outcomes of patients with coronavirus disease 2019 (COVID-19). JAMA Cardiol 2020;5:811.

16 Phua J, Weng L, Ling L, Li Weng LL, Egi M, et al. Intensive care management of coronavirus disease 2019 (COVID-19): challenges and recommendations. Lancet Respir Med 2020;8:506-17. 
17 Aziz S, Arabi YM, Alhazzani W, et al. Managing ICU surge during the COVID-19 crisis: rapid guidelines. Intensive Care Med 2020;46:1303-25.

18 Wang Y, Dong C, Hu Y, et al. Temporal changes of CT findings in 90 patients with COVID-19 pneumonia: a longitudinal study. Radiology 2020;296:E55-64.

19 Wu Z, McGoogan JM. Characteristics of and important lessons from the coronavirus disease 2019 (COVID-19) outbreak in China: summary of a report of 72314 cases from the chinese center for disease control and prevention. JAMA 2020. [Epub ahead of print: 24 Feb 2020].

20 Marrie TJ, Shariatzadeh MR. Community-acquired pneumonia requiring admission to an intensive care unit: a descriptive study. Medicine 2007;86:103-11.

21 Kuba K, Imai Y, Rao S, et al. A crucial role of angiotensin converting enzyme 2 (ACE2) in SARS coronavirus-induced lung injury. Nat Med 2005;11:875-9.

22 Pasqualetto MC, Secco E, Nizzetto M, et al. Stress cardiomyopathy in COVID-19 disease. Eur J Case Rep Intern Med 2020;7:001718.

23 Huang C, Wang Y, Li X, et al. Clinical features of patients infected with 2019 novel coronavirus in Wuhan, China. Lancet 2020.

24 Du Y, Tu L, Zhu P, et al. Clinical features of 85 fatal cases of COVID-19 from Wuhan. A retrospective observational study. Am J Respir Crit Care Med 2020;201:1372-9.

25 Weaver LK, Behrens EM. Weathering the storm: improving therapeutic interventions for cytokine storm syndromes by targeting disease pathogenesis. Curr Treatm Opt Rheumatol 2017;3:33-48.

$26 \mathrm{Hu} \mathrm{H}$, Ma F, Wei X, Hongde Hu F, et al. Coronavirus fulminant myocarditis saved with glucocorticoid and human immunoglobulin Eur Heart J 2020. doi:10.1093/eurheartj/ehaa190. [Epub ahead of print: 16 Mar 2020].

27 Ruan Q, Yang K, Wang W, et al. Clinical predictors of mortality due to COVID-19 based on an analysis of data of 150 patients from Wuhan, China. Intensive Care Med 2020;46:846-8.

28 Chen C, Zhou Y, Wang DW. SARS-CoV-2: a potential novel etiology of fulminant myocarditis. Herz 2020;45:230-2.

29 Tavazzi G, Pellegrini C, Maurelli M, et al. Myocardial localization of coronavirus in COVID-19 cardiogenic shock. Eur J Heart Fail 2020;22:911-5.

30 Fried JA, Ramasubbu K, Bhatt R, et al. The variety of cardiovascular presentations of COVID-19. Circulation 2020;141:1930-6.

31 Wang D, Hu B, Hu C, et al. Clinical characteristics of 138 hospitalized patients with 2019 novel coronavirus-infected pneumonia in Wuhan, China. JAMA 2020. doi:10.1001/ jama.2020.1585. [Epub ahead of print: 07 Feb 2020].

32 Sapp JL, Alqarawi W, Maclntyre CJ, et al. Guidance on minimizing risk of drug-induced ventricular arrhythmia during treatment of COVID-19: a statement from the Canadian heart rhythm Society. Can $J$ Cardiol 2020;36:948-51.

33 Ramanathan K, Antognini D, Combes A. Since January 2020 Elsevier has created a COVID-19 resource centre with free information in English and Mandarin on the novel coronavirus COVID- research that is available on the COVID-19 resource centre - including this for unrestricted research re-use, 2020: 19-21.

34 Brojakowska A, Narula J, Shimony R, et al. Clinical Implications of SARS-CoV-2 Interaction With Renin Angiotensin System: JACC Review Topic of the Week. J Am Coll Cardiol 2020;75:3085-95.

35 Danser AHJ, Epstein M, Batlle D. Renin-angiotensin system blockers and the COVID-19 pandemic: at present there is no evidence to abandon renin-angiotensin system blockers. Hypertension 2020;75:1382-5.

36 Sriram K, Insel PA. Risks of ACE inhibitor and ARB usage in COVID-19: evaluating the evidence. Clin Pharmacol Ther 2020;108:236-41.

37 Zhang X, Yu J, Pan L-Y, et al. ACEI/ARB use and risk of infection or severity or mortality of COVID-19: a systematic review and metaanalysis. Pharmacol Res 2020;158:104927.

38 Johri AM, Galen B, Kirkpatrick JN, et al. Ase statement on point-ofcare ultrasound during the 2019 novel coronavirus pandemic. J Am Soc Echocardiogr 2020;33:670-3.
39 Peng Q-Y, Wang X-T, Zhang L-N, et al. Findings of lung ultrasonography of novel corona virus pneumonia during the 20192020 epidemic. Intensive Care Med 2020;46:849-50.

40 Vetrugno L, Bove T, Orso D, et al. Our Italian experience using lung ultrasound for identification, grading and serial follow-up of severity of lung involvement for management of patients with COVID-19. Echocardiography 2020;37:625-7.

41 Marini JJ, Gattinoni L. Management of COVID-19 respiratory distress. JAMA 2020. doi:10.1001/jama.2020.6825. [Epub ahead of print: 24 Apr 2020].

42 Volpicelli G, Gargani L. Sonographic signs and patterns of COVID-19 pneumonia. Ultrasound J 2020;12:22.

43 Lichtenstein D. Lung ultrasound in the critically ill. Curr Opin Crit Care 2014;20:315-22.

44 Gargani L, Soliman-Aboumarie H, Volpicelli G, et al. Why, when, and how to use lung ultrasound during the COVID-19 pandemic: enthusiasm and caution. Eur Heart J Cardiovasc Imaging 2020;21:941-8.

45 Neskovic AN, Skinner H, Price S, et al. Focus cardiac ultrasound core curriculum and core syllabus of the European association of cardiovascular imaging. Eur Heart $J$ Cardiovasc Imaging 2018;19:475-81

46 Cardim N, Dalen H, Voigt J-U, et al. The use of handheld ultrasound devices: a position statement of the European association of cardiovascular imaging (2018 update). Eur Heart J Cardiovasc Imaging 2019;20:245-52.

47 Kirkpatrick JN, Mitchell C, Taub C, et al. Ase statement on protection of patients and echocardiography service providers during the 2019 novel coronavirus outbreak: endorsed by the American College of cardiology. J Am Soc Echocardiogr 2020;33:648-53.

48 Fischer EA, Kinnear B, Sall D, et al. Hospitalist-Operated compression ultrasonography: a point-of-care ultrasound study (HOCUS-POCUS). J Gen Intern Med 2019;34:2062-7.

49 Ryan D, Frohlich S, McLoughlin P. Pulmonary vascular dysfunction in ARDS. Ann Intensive Care 2014;4:28.

50 Ren B, Yan F, Deng Z, et al. Extremely high incidence of lower extremity deep venous thrombosis in 48 patients with severe COVID-19 in Wuhan. Circulation 2020;142:181-3.

51 Thachil J, Cushman M, Srivastava A, Jecko Thachil MD. A proposal for staging COVID-19 coagulopathy. Res Pract Thromb Haemost 2020;4:731-6.

52 Klok FA, Kruip MJHA, van der Meer NJM, et al. Incidence of thrombotic complications in critically ill ICU patients with COVID-19. Thromb Res 2020;191:145-7.

53 Magro C, Mulvey JJ, Berlin D, et al. Complement associated microvascular injury and thrombosis in the pathogenesis of severe COVID-19 infection: a report of five cases. Trans/ Res 2020;220:1-13.

54 Tang N, Li D, Wang X, et al. Abnormal coagulation parameters are associated with poor prognosis in patients with novel coronavirus pneumonia. J Thromb Haemost 2020;18:844-7.

55 Cui S, Chen S, Li X, et al. Prevalence of venous thromboembolism in patients with severe novel coronavirus pneumonia. J Thromb Haemost 2020;18:1421-4

56 Zhang L, Yan X, Fan Q, et al. D-dimer levels on admission to predict in-hospital mortality in patients with Covid-19. J Thromb Haemost 2020;18:1324-9

57 Thachil J, Tang N, Gando S, et al. ISTH interim guidance on recognition and management of coagulopathy in COVID-19. J Thromb Haemost 2020;18:1023-6.

58 Moores LK, Tritschler T, Brosnahan S, et al. Prevention, diagnosis and treatment of venous thromboembolism in patients with COVID-19: chest guideline and expert panel report. Chest J 2020.

59 Andreini D, Arbelo E, Barbato E, et al. ESC guidance for the diagnosis and management of CV disease during the COVID-19 pandemic. Available: https://www.escardio.org/Education/COVID19-and-Cardiology/ESC-COVID-19-Guidance

60 Tang T, Bidon M, Jaimes JA, et al. Coronavirus membrane fusion mechanism offers a potential target for antiviral development. Antiviral Res 2020;178:104792. 